Antje Dammel (Mainz)

\title{
Die schönen alten Formen ... \\ Grammatischer Wandel der deutschen Verbalflexion - Verfall oder Reorganisation?*
}

\begin{abstract}
Betrachtet man „Verfallserscheinungen“ des Verbalsystems wie Übergänge stark > schwach, so zeigt sich, dass hier weder Rezenz noch Verfall zu konstatieren ist. Mit diachroner und analytischer Tiefe offenbart sich ein gestaffelter, systematischer Komplexitätsabbau, der seine Hochphase im Frühneuhochdeutschen hat und sich schlecht mit der Passivität und Chaos implizierenden Verfallsmetapher verträgt: Reorganisation statt Dekadenz. Entwicklungen wie der präteritale Numerusausgleich (ich sang - wir sungen > ich sang - wir sangen) oder die Herausbildung der vereinfachten Ablautalternanz $\mathrm{X}-0-0$ sind nie nur Komplexitätsreduktion, sondern immer auch Systematisierung; sie bremsen Verfall. Dabei ist der Gewinn an Systematik i.d.R. nicht Normautoritäten geschuldet, sondern ihm liegen sprachsystematische, kognitive und frequenzielle Faktoren zugrunde.
\end{abstract}

\section{Einleitung}

Die laienlinguistisch-wertende Frage nach Sprachverfall haben die Organisator/innen der IDS-Jahrestagung mit dem Untertitel Sprachdynamik - Spracbvariation - Sprachwandel schon selbst negativ beantwortet und damit sozusagen Eulen nach Athen getragen. Linguist/innen wissen, dass Sprachverfallsklagen hochgradig sozial aufgeladen sind, dass entlang von konservativen versus innovativen grammatischen Varianten soziale Grenzen konstruiert wurden und werden. Man kann das als gegeben hinnehmen. Eine andere Möglichkeit, die hier verfolgt werden soll, ist es, solche Grenzziehungen offenzulegen und das sozial motivierte laienlinguistische Interesse an sprachlicher Variation als Aufhänger für Aufklärungsarbeit zu nutzen; Laien zuzutrauen, sich auf der Objektebene mit Problemen des Sprachwandels auseinanderzusetzen und so eine differenziertere Sicht auf Sprachwandel zu gewinnen. Dieser Beitrag demonstriert das an einem Modellfall, der notorisch von Verfallsklagen betroffen ist: dem „Aussterben“ starker Verben (Kap. 2). Er richtet sich dabei nicht direkt an Laien - dafür wären andere Publikationsorte geeigneter -, sondern versteht sich als eine „Gebrauchs-

* Ich danke Jessica Nowak dafür, dass sie mir ihr umfangreiches Korpus historischer Grammatiken sowie vorab Ergebnisse ihrer Dissertation „Zur Legitimation einer 8. Ablautreihe“ (i.Vorb.) zur Verfügung gestellt hat und ganz besonders dafür, dass sie jederzeit für ein Gespräch über Ablaut zu begeistern ist. 
anweisung für Aufklärer“, die durchspielt, welche Argumente man heranziehen könnte. Dafür ist das IDS-Jahrbuch als eine Schnittstelle zwischen Fachwissenschaft und Öffentlichkeit nicht der schlechteste Ort.

Dieses Vorhaben bringt mit sich, dass die Metapher Verfall hier bewusst ernst und wörtlich genommen wird, nicht auf ihre wertenden Konnotationen hin, sondern als analytischer Ansatzpunkt, der sich an gegebenen Entwicklungen verifizieren lässt (siehe Tab. 1): Was müsste mit einer Sprache passieren, um von Sprachverfall sprechen zu können? Sind entsprechende Prozesse in der Diachronie der deutschen Verbflexion festzustellen?

\begin{tabular}{|c|c|}
\hline Wertende Konnotationen & Analytische Ansatzpunkte \\
\hline Wildwuchs: chaotisch, ungeregelt & Gestaffelt, prinzipiengesteuert? \\
\hline $\begin{array}{l}\text { Passivität, Dekadenz: Sprecher/innen } \\
\text { lassen ihre Sprache verlottern }\end{array}$ & $\begin{array}{l}\text { Gleichgerichte Aktivität von } \\
\text { Sprecher/innen reorganisiert das } \\
\text { System }\end{array}$ \\
\hline $\begin{array}{l}\text { Verarmung } \\
\text { - durch Verlust obligatorischer } \\
\text { Distinktionen } \\
\text { durch Verlust synthetischer zu- } \\
\text { gunsten analytischer Strukturen }\end{array}$ & $\begin{array}{l}\text { Reduktion morphologischer } \\
\text { Komplexität } \\
\text { - Komplexitätsreduktion kann } \\
\text { Systematisierung implizieren: } \\
\text { Wo wird Komplexität erhalten, } \\
\text { wo nicht? } \\
\text { - Differenzierung nach Inventar- } \\
\text { ebene (Anzahl der Einheiten/ } \\
\text { Optionen) und Systemebene } \\
\text { (Komplexität der Anwendung) }\end{array}$ \\
\hline $\begin{array}{l}\text { Hier und jetzt } \\
\text { Früher war das Deutsche intakt, } \\
\text { gegenwärtig geht es bergab }\end{array}$ & $\begin{array}{l}\text { Langzeitperspektive } \\
\text { Kontinuität des Phänomens, } \\
\text { Einbettung in übergreifende } \\
\text { Zusammenhänge }\end{array}$ \\
\hline
\end{tabular}

Tab. 1: Perspektiven auf Sprachverfall

Ausgehend von diesen Vorüberlegungen wird Wandel im Bereich der starken Verbflexion, der laienlinguistisch als rezente Verfallserscheinung gehandelt wird, aus der Langzeitperspektive untersucht und in übergreifende Systemzusammenhänge eingebettet. Es wird sich zeigen, dass wir es nicht mit Verfall, sondern mit prinzipiengesteuertem Wandel zu tun haben, der seinen Schwerpunkt in der frühneuhochdeutschen (fnhd.) Phase (13501650) hat. Gerade das Fnhd. eignet sich sehr gut für Aufklärungsarbeit, weil in dieser Zeit eine tiefgreifene Umstrukturierung auf der morphologischen Ebene stattfand und viele der heutigen grammatischen Zweifelsfälle angelegt wurden (vgl. Klein 2011; zum Genitiv z.B. Szczepaniak in diesem Band). Mit der fnhd. Phase beginnt auch die Vertikalisierung des Deutschen, bei der Erwartungen an eine normierte Standardsprache entstehen, und es for- 
miert sich eine Tradition der Grammatikographie, die auf Wandel reagiert, ihn aufgreift und bis ins 20. Jahrhundert auch wertet - ihn damit aber nur seltenst beeinflusst (vgl. Hundt 2009 zu den verschiedenen Determinanten der Normkonstitution). Dazu kommt mit dem Buchdruck eine Umwälzung im Bereich der Medien, die Vereinheitlichungen im grammatischen System mitbedingt, die syntaktische Komplexität generiert und die so das Argument „die Medien bedingten Sprachverfall“ als Pauschalisierung entlarvt.

\section{Ein (Ver-?)Fallbeispiel: Flexion der starken Verben}

Einen Abgesang auf den Ablaut der starken Verben stimmt schon J. Grimm, der Erfinder des Begriffs Ablaut, an:

[...] so hat sich dagegen die herliche und dauerhafte natur des deutschen verbums fast nicht verwüsten lassen, und von ihr gehn unzerstörbar klang und klarbeit in unsere sprache ein. die grammatiker, welche ihre sprachkunde auf der oberfläche, nicht in der tiefe schöpften, haben zwar alles gethan, um den ablaut, der die edelste regel deutscher conjugation bildet, als ausnahme, die unvollkommene flexion als regel darzustellen, so dasz dieser der rang und das recht zustehe jene allmälich einzuschränken, wo nicht gar aufzuheben. füblt man aber nicht, dasz es schöner und deutscher klinge zu sagen buk, wob boll (früher noch besser wab ball) als backte webte bellte, und dasz zu jener form die participia gebacken gewoben gebollen stimmen? im gesetze des ablauts gewahre ich [...] den ewig schaffenden wachsamen sprachgeist, der aus einer anfänglich nur phonetisch wirksamen regel mit dem heilsamsten wurf eine neue dynamische gewalt entfaltete, die unserer sprache reizenden wechsel der laute und formen zuführte. es ist sicher alles daran gelegen ihn zu behaupten und fortwährend schalten zu lassen. (Grimm [1847] 1864, S. 340; Hervorhebungen AD)

Bei Grimm findet sich ein häufig realisierter Topos der Sprachkritik, die Ästhetisierung und Aufwertung des Archaischen (kursive Markierung), Ablaut wird als zu wahrendes Kulturgut dargestellt; nicht zufällig geht dies mit nationalistischen Anklängen einher (Unterstreichung). Gleichzeitig wird aus dem Zitat auch deutlich, dass die frühen Grammatiker des 17./18. Jahrhunderts ein weitaus pragmatischeres Verhältnis zum Ablaut hatten und mit Listen und Beispielparadigmen Lernschwierigkeiten beheben wollten, daher auch Bezeichnungen wie „ungleichfließend“, „unordentlich“ für die starke Konjugation (z.B. Gueintz 1978 [1641], S. 68). Dieselbe Ästhetisierung des Konservativen finden wir noch in modernen Sprachratgebern wie z.B. Schneider (2009), vgl. auch Corr (2013).

Ja, auch jüngere Leute können Sätze wie diesen noch verstehen: «Was hülfe es dem Menschen, wenn er die ganze Welt gewönne und nähme doch Schaden an seiner Seele?» (Matthäus 16, 26). [...] - wer immer sein Publikum gewinnen will, muss wohl in Rechnung stellen, dass die schönen alten Formen (oh, glömme doch ein Feuer!) auf die meisten Adressaten archaisch wirken, wenn nicht manieriert. (Schneider 2009, S. 11, Hervorhebungen AD) 
Schneiders Äußerung teilt mit anderen sprachkritischen Aussagen nicht nur den häufig bemühten Topos „die Jugend ist schuld“", sondern auch den Aspekt der Pauschalisierung. Denn Konjunktivformen starker Verben fallen nicht alle gleichermaßen als archaisch auf, vgl. etwa ließe und gäbe, die unauffällig und gebräuchlich sind.

Es ist also eine häufig vorgebrachte Verfallsklage, dass die starken Verben langfristig aussterben, vor allem indem sie zur schwachen Flexion übergehen. Analytisch betrachtet wäre dies eine Systemvereinfachung, ein Abbau flexionsmorphologischer Komplexität. Die diversifizierten Flexionsmuster der starken Verben sind schwer zu lernen und leisten auf den ersten Blick nichts anderes als das schwache Dentalsuffix. Aus der Langzeitperspektive und differenziert nach Inventar- und Systemebene zeigt sich, dass diese Komplexität äußerst resistent und funktional ist.

\subsection{Inventarebene}

Im Inventar ist tatsächlich ein deutlicher Rückgang der starken Flexion zu verzeichnen (siehe Abb. 1); doch ist dieser in erster Linie nicht wie häufig angenommen dem Übergang zur schwachen Klasse geschuldet (62 Vb.), sondern vor allem durch Schwund bedingt (160 Verben, z.B. ahd. quedan ,sprechen'). Worttod betrifft schwache Verben gleichermaßen, nur ist hier die Grundgesamtheit wesentlich größer und die Klasse voll produktiv. Abbildung 1 zeigt auch, dass ausnahmsweise neue starke Verben entstehen können, z.B. im Althochdeutschen (Ahd.) die Entlehnung scriban ,schreiben und zum Neuhochdeutschen hin z.B. das ehemals schwache Verb preisen, das sich ebenfalls dem mitgliederstarken Muster $e i-i:-i$ : anschließt.

\begin{tabular}{rcc} 
AHD & MHD & NHD \\
\hline $349(100 \%)$ & $339(98 \%)$ & $(48 \%)$ \\
$>$ schwache Vb.: & -8 & > schwache Vb.: -54 \\
ausgestorben: -41 & ausgestorben: -119 \\
neu/aus schw. Vb.: +39 & aus schw. Vb.: +3
\end{tabular}

Abb. 1: Rückgang starker Verben seit dem Althochdeutschen ${ }^{1}$

Betrachtet man die wenigen Übergänge zur schwachen Flexion genauer, so zeigt sich erstens, dass deren Hochphase im 16. Jahrhundert zu verorten und bereits seit dem 18. Jahrhundert eine Sättigungstendenz eingetreten ist (vgl. auch Kupietz in diesem Band). Und zweitens streuen die Übergänge nicht willkürlich, sondern bilden eine $s$-Kurve, die sich vorausberechnen lässt und die bei zahlreichen Sprachwandelerscheinungen (vgl. Zuraw 2003), aber

Zahlen nach Augst (1975, S. 255), Visualisierung nach Wegera/Waldenberger (2012, S. 178). 
auch Naturphänomenen wie dem radioaktiven Zerfall, zu beobachten ist. Selbst „Verfall“ verläuft also nicht chaotisch, sondern lässt sich regelhaft beschreiben.

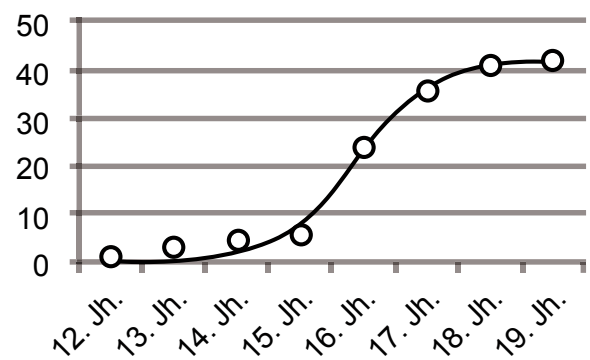

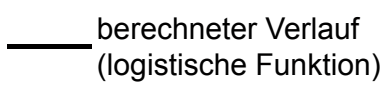

Schwache Erstbelege in

○ Prät./Part.II (DWB, Paul) kumulativ

Abb. 2: Übergänge starker Verben in die schwache Flexion (Kurve nach Best 2003, S. 13, Zahlen nach Faust 1980, S. 400-404)

Ein wichtiger Gegenspieler der Regularisierung ist hohe Gebrauchsfrequenz. Formen, die wir häufig nutzen, und das ist bei den meisten verbliebenen starken Verben der Fall, memorieren wir gut und speichern wir ganzheitlich ab. Dass starke Formen kürzer und deutlicher unterschieden sind als schwache, ist bei häufigem Gebrauch vorteilhaft für Produktions- wie Perzeptionsseite. Das gilt in besonderem Maße für Funktionsverben, die noch stärkere Irregularitätsgrade als starke aufweisen, z.B. haben, das diachron aus der regulären schwachen Klasse ausgeschert ist und unikale Flexionseigenschaften entwickelt hat. ${ }^{2}$ Das erlaubt die Prognose, dass es unter den bestehenden Bedingungen im Deutschen nicht zu einer vollen Vereinheitlichung (nur schwache Verben) kommen wird; unterschiedliche Grade von (Ir-)Regularität werden sich in verschiedenen Gebrauchsfrequenzbereichen halten (z.B. Werner 1987).

Für die Inventarebene lässt sich damit festhalten, dass diachron zwar ein deutlicher Verlust starker Verben zu verzeichnen ist, dieser aber heute stagniert und dass die noch vorhandenen starken Verben, die meist fester Bestandteil des Grundwortschatzes sind, auf absehbare Zeit erhalten bleiben (vgl. z.B. auch Augst 1975).

\subsection{Systemebene}

Betrachtet man die Systemebene aus der diachronen Langzeitperspektive, so wird Ablaut, das Hauptkennzeichen starker Verben, eher systematisiert und integriert als abgebaut. Tabelle 2 gibt einen Überblick über die relevanten Prozesse, die im Folgenden schrittweise skizziert werden.

2 Nach Augst (1975, S. 258) flektieren von 4000 Verbsimplizia im Lexikon 3811 (95,3\%) schwach, $169(4,2 \%)$ stark und $20(0,5 \%)$ anderweitig irregulär (z.B. haben, müssen). Von den im Fließtext vorkommenden Verbformen sind dagegen durchschnittlich gleich viele stark und schwach (je 41\%) und 18\% irregulär. 


\begin{tabular}{|c|c|}
\hline Entwicklung des Ablauts & Illustration \\
\hline $\begin{array}{l}\text { IDE } \\
\text { Ablaut ist Begleiterscheinung zu } \\
\text { Akzentverhältnissen und Affixen }\end{array}$ & $\begin{array}{ll}\text { Inf. } & \text { 1.Pl. Perfekt } \\
* k e ́ l b-o n o m & * k e-k l b \text {-mé } \\
\text { Normalstufe } & \text { Schwundstufe }\end{array}$ \\
\hline $\begin{array}{l}\text { GERM, AHD } \\
\text { Vereinfachung und Systematisierung } \\
\text { nach Stammauslaut (gut lernbar aber nicht } \\
\text { auf jedes neue Verb anwendbar); } \\
\text { Funktionalisierung: Tempus (Modus/ } \\
\text { Numerus/Person) (Nebenmarker zu } \\
\text { distinkten Suffixen) }\end{array}$ & $\begin{array}{ll}\text { 1.Pl.Präs. } & \text { 1.Pl.Prät. } \\
\text { helf-emēs } & \text { bulf-um }\end{array}$ \\
\hline $\begin{array}{l}\text { MHD } \\
\text { Ablaut wird Hauptmarker } \\
\text { (Nebensilbenreduktion) }\end{array}$ & helf-en \\
\hline $\begin{array}{l}\text { FNHD } \\
\text { Aufsplitterung der Ablautreihen } \\
\text { (Lernbarkeit sinkt) } \\
\text { Reduktion und Systematisierung der } \\
\text { Ablautstufen: Tempusausdruck }\end{array}$ & $\begin{array}{l}7>40 \text { Ablautmuster } \\
\text { helfen-half }\end{array}$ \\
\hline $\begin{array}{l}\text { (F)NHD } \\
\text { frequenzgesteuerte Umschichtungen, } \\
\text { Entstehung neuer Subregularitäten wie X-o-o } \\
\text { Erhalt bei ausreichender Frequenz bzw. } \\
\text { „Inseln der Verlässlichkeit““ (für starke Verben } \\
\text { typischen phonologischen Strukturen) }\end{array}$ & $\begin{array}{l}\text { A-B-A, A-B-C > A-B-B } \\
+++ \text { Gebrauchsfrequenz --- } \\
\text { Einzelverben Großgruppen } \\
\text { z.B. Stamm auf - } i \mathrm{NC}\end{array}$ \\
\hline
\end{tabular}

Tab. 2: Entwicklung des Ablauts starker Verben (Überblick)

Ablaut beginnt im Vor-Indoeuropäischen als phonetische Alternation, die durch Akzentunterschiede und andere kombinatorische Lautwandelprozesse verursacht ist (z.B. Mailhammer 2007a, S. 15-19 mit Lit.). Im Indoeuropäischen (Ide.), das man etwa um $4000 \mathrm{v}$. Chr. ansetzt, ist die Ablautalternation zwar verfestigt, aber noch immer eine bloße Begleiterscheinung zu Akzentverhältnissen und Affixen. Exemplarisch lässt sich das an dem Formenpaar Infinitiv *kélb-onom ,helfen' - 1.Pl.Perfekt*ke-klb-mé zeigen. Die lexikalische Wurzel ist - $k \_l b$ - Im Infinitiv liegt der Akzent auf der Wurzel, hier steht die Vollstufe $e$; im Perfekt, bei dem das Suffix betont ist, liegt die Wurzel in der Akzentsenke. Deshalb zeigt der Ablaut hier eine Schwundstufe. Der ide. Ablaut ist also in vielen Fällen durch seine Umgebung vorhersagbar.

Im Ide. gab es eine große Anzahl verschiedener Ablautmuster. Das ändert sich zum Germanischen (Germ.) hin: Hier wird Ablaut stark vereinfacht, systematisiert und funktionalisiert (siehe Tab. 3, Reihen I-V). 


\begin{tabular}{|c|c|c|c|c|c|}
\hline Reihen & $\begin{array}{r}\text { Stufen } \\
\text { Funktionen }\end{array}$ & $\begin{array}{l}1 \\
\text { Inf., Präs., } \\
\text { Konj. I }\end{array}$ & $\begin{array}{l}2 \\
\text { Prät.Ind. } \\
\text { 1./3.Sg. }\end{array}$ & $\begin{array}{l}3 \\
\text { Prät.Ind. } \\
\text { Pl., 2.Sg. } \\
\text { Konj. II }\end{array}$ & $\begin{array}{l}4 \\
\text { Part. II }\end{array}$ \\
\hline \multirow{5}{*}{$\begin{array}{l}\text { I-V } \\
\text { ide. } \\
\text { Vorläufer }\end{array}$} & $-i$ & \multirow{5}{*}{$\begin{array}{l}\text { Normalstufe } \\
\text { idg., germ. } \boldsymbol{e}\end{array}$} & \multirow{5}{*}{$\begin{array}{l}\text { Abtönstufe } \\
\text { ide. } \boldsymbol{o}>\text { germ. } a\end{array}$} & \multirow{3}{*}{\multicolumn{2}{|c|}{$\begin{array}{l}\text { Schwundstufe } \\
\text { I+II: ide., germ. Null } \\
\text { III: } \quad \text { ide. Null > germ. } u\end{array}$}} \\
\hline & $\mathrm{II} \quad-u$ & & & & \\
\hline & III _N/L+C & & & & \\
\hline & $\mathrm{IV} \quad{ }_{-} \mathrm{N} / \mathrm{L}$ & & & \multirow{2}{*}{$\begin{array}{l}\text { Dehnstufe } \\
\text { ide. } \overline{\boldsymbol{e}}>\text { germ. } \bar{a}\end{array}$} & \\
\hline & $\mathrm{V} \quad \mathrm{C}^{(\neq \mathrm{N} / \mathrm{L})}$ & & & & $\begin{array}{l}\text { Normalstufe } \\
\text { ide., germ. } e\end{array}$ \\
\hline $\begin{array}{l}\text { VI } \\
\text { (Germ.) }\end{array}$ & $a \mathrm{C}(\mathrm{C})$ & $a$ & \multicolumn{2}{|c|}{ germ. $\bar{o}>$ ahd. $u o$} & $a$ \\
\hline $\begin{array}{l}\text { VII } \\
\text { (Ahd.) }\end{array}$ & $\begin{array}{l}(<\text { reduplizie- } \\
\text { rende Verben })\end{array}$ & $\begin{array}{l}a, \bar{a}, e i ; \\
\text { ou, ō, uo }\end{array}$ & \multicolumn{2}{|l|}{ ahd. ie } & $\begin{array}{l}a, \bar{a}, e i ; \\
\text { ou, } \bar{o}, \text { uo }\end{array}$ \\
\hline
\end{tabular}

Tab. 3: Das altgermanische Ablautsystem $(\mathrm{N}=$ Nasal, $\mathrm{L}=$ Liquid, $\mathrm{C}=$ Konsonant, $\mathrm{V}=$ Vokal $)$

Die Vereinfachung besteht vor allem darin, dass von den vielen Mustern des Ide. im Wesentlichen nur dieses überlebt: $e_{-0-N u l l}$ (mit kleinen Abweichungen in Reihen IV und V). Ablaut emanzipiert sich dabei vom Akzent, der nun fest auf der Stammsilbe liegt. Systematisierung und Funktionalisierung zeigen sich daran, dass die Ablautstufen, also die Teile eines Musters, jetzt fest in die Anzeige verbaler Kategorien eingebunden werden. ${ }^{3}$ Dieses System ist relativ leicht lernbar: Es gibt nur wenige Alternanzmuster, die Zuordnung ist aufgrund der phonologischen Struktur des Stammes weitgehend vorhersagbar (vgl. z.B. Mailhammer 2007b). Indem der Ablaut diesem System gehorcht, ist er ab dem Germ. an der Markierung von Tempus beteiligt, alterniert aber auch für weitere Kategorien, nämlich Modus, Numerus und im Westgerm. sogar für Person (2.Ps.Sg.Prät.Ind.), vgl. die folgenden althochdeutschen Formen von ,helfen' aus Ablautreihe III:

Stufe 1

(ehem. Vollstufe, ide. e)

helfemēs 1.Pl.Präs.Ind.

\section{Stufe 2}

(ehem. Abtönung, ide. $o>$ germ. $a$ )
Stufe 3,4

(ehem. Schwundstufen, germ. Sprossvokal $u$ )

half 1./3.Sg.Prät.Ind. hulf-i 2.Sg.Prät.Ind.

bulf-um 1.Pl.Prät.Ind.

bulf-im 1.Pl.Prät.Konj.

gi-holfan Part. II (germ. $u>o$ vor $a)$

Dabei wird das alte Aspektsystem des Ide. (+/- perfektiv) als Tempussystem uminterpretiert, alte Perfekt- werden zu neuen Präteritalformen (vgl. Ringe 2006, Kap. 3.3.1). Als mögliche Ursache für diese Reorganisation und Vereinfachung kommt früher Sprachkontakt in Betracht (Stedje 1987; Mailhammer 2007a), dessen genaue Konstellation aber zu weiten Teilen spekulativ bleiben muss. 
Dabei zeigt sich aber auch, dass Ablaut noch immer nicht ganz selbstständig ist und im Vergleich zu den noch gut unterschiedenen Suffixen den Status eines Nebenmarkers hat (Wurzel 1996). Das ändert sich entscheidend zum Mittelhochdeutschen (Mhd.) hin:

Stufe 1

belf-en 1.Pl.Präs.Ind.
Stufe 2

half 1./3.Sg.Prät.Ind.
Stufe 3,4

$\begin{array}{ll}\text { bülf-e } & \text { 2.Sg.Prät.Ind. } \\ \text { bulf-en } & \text { 1.Pl.Prät.Ind. } \\ \text { bülf-en } & \text { 1.Pl.Prät.Konj. } \\ \text { ge-bolfen } & \text { Part. II }\end{array}$

Durch die Reduktion aller unbetonten vollen Vokale zu $e[ə]$ verlieren die Suffixe an Distinktivität, und bei einigen Formen rückt dadurch der Ablaut zum Hauptmarker auf, der die Information alleine trägt, z.B. bei dem Paar wir helfen - bulfen (1.Pl. Präsens vs. Prät.). Aus der Perspektive des Systems wird der Ablaut damit keineswegs abgebaut, sondern gewinnt sogar diachron an Funktionalität.

Im Fnhd. setzen dann Prozesse ein, die den Ablaut auf der Ebene der verschiedenen Muster (Ablautreihen) verkomplizieren, auf der Ebene der Teile eines Musters (Ablautstufen) aber vereinfachen und systematisieren: Während das ursprüngliche System mit seinen max. sieben Ablautreihen und den typischen konsonantischen Strukturen der Wurzel noch leicht lernbar war, verkompliziert sich zum Nhd. die Lage. Aus sieben werden knapp 40 Ablautmuster. ${ }^{4}$ Schuld daran ist zum einen Lautwandel, der abhängig vom Kontext unterschiedlich greift: Die fnhd. Dehnung kurzer Vokale in offener Tonsilbe hat z.B. bei Verben mit stimmhaftem Dentalplosiv gewirkt (meiden $-m[\mathrm{i}:] d-\operatorname{gem}[\mathrm{i}:] d e n)$, bei stimmlosem aber nicht (reiten $-r[\mathrm{I}] t t-$ ger[I]tten). Das Verb kommen (< ahd. queman) hat sich gänzlich aus seiner Ablautreihe IV abgesetzt und sich zum Einzelgänger entwickelt, indem im Präsens der ehem. Labial im Anlaut qu- [kv] den folgenden Vokal $e$ zu $o$ irregulär gerundet hat. Aber auch morphologische Prozesse haben zu Abspaltungen geführt: Bei Verben der Ablautreihe III hat sich im Präteritum normalerweise der Sg.-Vokal a durchgesetzt: ich half/sang - wir hulfen/sungen $\rightarrow$ half, halfen/sang, sangen. Nur werden hat (wohl unter Einfluss der an Frequenz gewinnenden Konj.-Form würde) den Pluralvokal zu heutigem wurde generalisiert und ward im 19. Jahrhundert aufgegeben. Auf ähnliche Weise entstanden zahlreiche neue Ablautmuster, die die Lernbarkeit des Systems erschweren.

Vgl. die Übersicht der Duden-Grammatik (Duden 1998, S. 127), die im Folgenden die Basis für alle Mengenangaben zum Ablaut nhd. starker Verben bildet. Spätere Auflagen systematisieren die Alternanzmuster nicht mehr so kompakt. 
Dieser Aufsplitterung auf der Ebene der Muster steht eine Vereinfachung der Stufen entgegen. Der an Numerus gekoppelte Ablaut im Präteritum wird ausgeglichen (so genannter präteritaler Numerusausgleich), in den Dialekten südlich der Mainlinie gefolgt vom gänzlichen Verlust des Präteritums:

mhd.

fnhd.

Dialekte etwa südl. der Mainlinie

$\begin{array}{cccc}\text { belfen } & \text { balf } & \text { bulfen } & \text { geholfen } \\ \text { belfen } & \text { half, halfen } & --- & \text { geholfen } \\ \text { belfe } & -\ldots-\ldots & & g(e) \text { bolfe }\end{array}$

Bei diesem Ausgleichprozess gewinnt im Deutschen teils der Sg.-, teils der Pluralvokal, was zu unterschiedlich differenzierten Alternationstypen führt: ABB, ABC und ABA (Solms 1984; Hempen 1988; Nübling 1998)..$^{5}$

\begin{tabular}{|c|c|c|c|c|c|}
\hline Reihe I: & $\begin{array}{l}\text { Präs. } \\
\text { reiten }\end{array}$ & $\begin{array}{l}\text { Prät.Sg. } \\
\text { reit }\end{array}$ & $\begin{array}{l}\text { Prät.Pl. } \\
\text { ritten }\end{array}$ & $\begin{array}{l}\text { Part.Perf. } \\
\text { geritten }\end{array}$ & $\begin{array}{l}\text { Alternationstyp } \\
\rightarrow \text { A-B-B } \\
\text { (*A-A-B reit hätte Tempus nivelliert) }\end{array}$ \\
\hline & elfen & half & thenten & gebolfen & $\rightarrow$ A-B-C \\
\hline Reihe V: & geben & th & gâben & gegeben & $\rightarrow$ A-B-A \\
\hline
\end{tabular}

Ein Hauptauslöser für diesen Vereinfachungsprozess, der sich vom 13. bis ins 18. Jahrhundert zieht und seine Hochphase im 16. Jahrhundert hat, ist die Frequenzzunahme des Perfekts auf Kosten des Präteritums, weil ersteres zunehmend den Funktionsbereich des Prät. übernimmt (Dentler 1998). Während vor 1400 in Texten noch das Präteritum dominiert (61\% Prät. : 39\% Part. II), ist um 1650 das Verhältnis zugunsten des Perfekts gekippt (25\% Prät. : 75\% Part. II) (Solms 1984, S. 311).

Man könnte den präteritalen Numerusausgleich als Abbau von Distinktionen und damit als Verfallserscheinung werten. Aber auch hier verläuft die Vereinfachung nicht zufällig, sondern hat System. Es entsteht z.B. nie ein Muster AAB, das in Reihe I phonologisch möglich gewesen wäre, aber den Tempusausdruck gefährdet hätte (Enger 1994). Auch verläuft der Ausgleich auf interessante Weise gestaffelt (Tab. 4): Schon vor dem 15. Jahrhundert wird das Sonderflexiv der 2.Ps.Sg.Prät. ausgeglichen ( $d u$ bülfe $>$ du balfst), dann erst folgt Numerus im Prät. (ich half - wir hulfen > halfen), was zu einer eindeutigen Koppelung des Ablauts an Tempus führt.

Andere germanische Sprachen haben andere Präferenzen (vgl. z.B. Durrell 2001; Hempen 1988). Im Niederländischen führt der Numerusausgleich z.B. zu sehr regulären Ablautmustern $(80 \% \mathrm{ABB})$ mit hoher Mitgliederzahl, die auch schwache Verben aufgenommen haben (z.B. zenden - zond-gezonden, senden). 


\begin{tabular}{|c|c|c|c|c|}
\hline \multicolumn{2}{|c|}{ Prät. } & $\begin{array}{l}\text { MHD } \\
\text { unsystematisch }\end{array}$ & $\begin{array}{l}\text { FNHD 15. Jh. } \\
\text { Numerus }\end{array}$ & $\begin{array}{l}\text { (F)NHD 16.-19. Jh. } \\
\text { Tempus }\end{array}$ \\
\hline \multirow{3}{*}{ Sg. } & 1. & half & balf & half \\
\hline & 2. & bülf-e $>$ bülf-est & balf-est & half-(e)st \\
\hline & 3. & half & half & half \\
\hline \multirow{3}{*}{ Pl. } & 1. & bulf-en & hulf-en & balf-en \\
\hline & 2. & bulf-et & bulf-et & balf- $(e) t$ \\
\hline & 3. & bulf-en & bulf-en & balf-en \\
\hline
\end{tabular}

Tab. 4: Systematisierung des Ablauts zum Tempusausdruck

Diese Staffelung gehorcht einer Generalisierung, die aus der Sprachtypologie bekannt ist, dem so genannten Relevanzprinzip (Bybee 1985; Nübling/ Dammel 2004). Dieses Prinzip besagt, dass Informationen, die das Verb stark in seiner Bedeutung beeinflussen, auch formal stärker mit dem Verb fusionieren sollten. Das ist beim Ablautausgleich der Fall: Die Informationen, die „nur“ Kongruenzkategorien sind und nicht das Verb direkt betreffen (Person, Numerus), werden aus dem Stamm ausgelagert und heute über Affixe und Pronomen markiert. Dadurch konzentriert sich Ablaut auf die für das Verb relevante Information Tempus. ${ }^{6}$

Nicht nur starke, auch schwache Verben wurden vereinfacht: Die ehemals knapp 200 Verben umfassende Mischklasse der Rückumlautverben, die durch einen Umlaut-Vokalwechsel und ein Dentalsuffix gekennzeichnet war (z.B. mhd. k.üssen - kuste ), wird in der fnhd. Phase bis auf wenige Verben abgebaut (z.B. Dammers/Hoffmann/Solms 1988, IS 160-164). Der Effekt ist eine klare Abgrenzung der beiden Großklassen stark und schwach, die weitere Übertritte hemmt, indem sie das Bewusstsein für zwei distinkte Klassen stärkt (Dammel 2010, S. 113-133).

Wenn starke Verben zur schwachen Flexion übertreten, geschieht auch das im Deutschen nicht willkürlich, sondern gestaffelt nach einer gerichteten Implikation (Abb. 3, verändert nach Bittner 1996; vgl. aber auch schon Adelung 1782, S. 819; für eine formale Modellierung siehe Clahsen et al. 2002). Die Schwächung starker Verben beginnt mit dem Abbau der Imperativhebung (Milk! > Melk(e)!), sofern diese vorhanden war. Es folgt die so genannte Wechselflexion zwischen 2./3.Ps.Sg. und dem übrigen Präsens sie milk.t $>$ melkt, dann schwanken die starken Präteritalformen im Indikativ und Konjunktiv molk $>$ melkte. Am stabilsten ist im Deutschen das Part. II gemolken.

Selbst im Afrikaans, der germanischen Sprache mit der sprachkontaktbedingt stärksten Deflexion, verlief der Abbau geordnet nach Relevanz: Numerus und Person wurden lange vor Tempus aufgegeben (siehe Dammel 2010, S. 107 mit Lit.). 


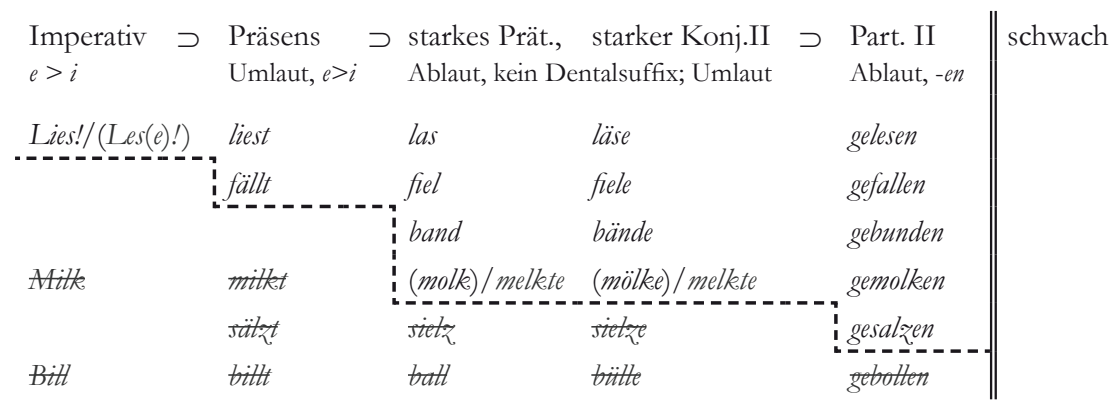

Abb. 3: Hierarchie des Vergessens starker Formen (nach Bittner 1996)

Diese Hierarchie gilt zwar nicht ausnahmslos, ist aber eine starke Generalisierung, die sich schon für das Fnhd. und ältere Nhd. aus Textkorpora und Aussagen der Grammatiker erschließen lässt (Nowak i.Vorb.). Die Staffelung kann man so interpretieren, dass sich hier Stammalternation schrittweise aus den Kategorien Person und Modus zurückzieht und auf den Tempusausdruck konzentriert, an dem, zumindest in konzeptioneller Mündlichkeit, das Perfekt heute am stärksten beteiligt ist.

Die vorgestellte Skala deckt aber im Spannungsfeld starker und schwacher Verben noch nicht alles ab, was System hat. Es lassen sich auch innerhalb der starken Verben, also solange Vokalwechsel erhalten bleiben, unterschiedliche Grade der Reorganisation und Regularisierung beobachten. Die nhd. ca. 40 Alternanzmuster starker Verben sind nicht gleich stark differenziert: Manche unterscheiden Prät. und Part. II durch einen gesonderten Vokal (belfen - half - geholfen $\mathrm{ABC}$, fahren - fuhr - gefahren $\mathrm{ABA}$ ), andere nicht (fliegen - flog - geflogen ABB). Letztere stehen damit dem Typus der schwachen Verben (Dentalsuffix in Prät. und Part. II) näher. Einige Alternanzmuster, besonders des Typs ABB, haben viele Mitglieder, unter denen auch selten gebrauchte Verben sind, z.B. $e i-i-i$ (streiten, schreiten) mit 23 Verben. Andere Muster sind nur durch ein Verb vertreten (z.B. $o-a:-o$ durch kommen) - meist ein sehr häufig gebrauchtes. Der Differenzierungsgrad von Alternanzmustern und deren Mitgliederstärke korrelieren also stark mit Gebrauchshäufigkeiten, siehe hierzu Abbildung 4.

Abbildung 4 zeigt links das Type-Token-Verhältnis für die drei abstrakten Distinktionstypen, rechts für ausgewählte konkrete Alternanzmuster: ABB hat viele Mitglieder mit durchschnittlich geringerer Gebrauchsfrequenz, während sich bei $\mathrm{ABC}$ und besonders $\mathrm{ABA}$ das Verhältnis umkehrt. In letzterem Typ konnten sich diachron fast nur hochfrequente Verben halten, die heute Kleingruppen oder Einzelalternanzen bilden (siehe e(:) - a(:) - e(:), geben, ganz rechts in Abb. 4). Hier besteht bei einem Rückgang der PräteritumVerwendung keine Vokalalternanz mehr, wodurch der Weg zum schwachen Verb besonders kurz ist: backen - $>$ gebacken (ABA, vgl. Solms 1984, S. 323). 


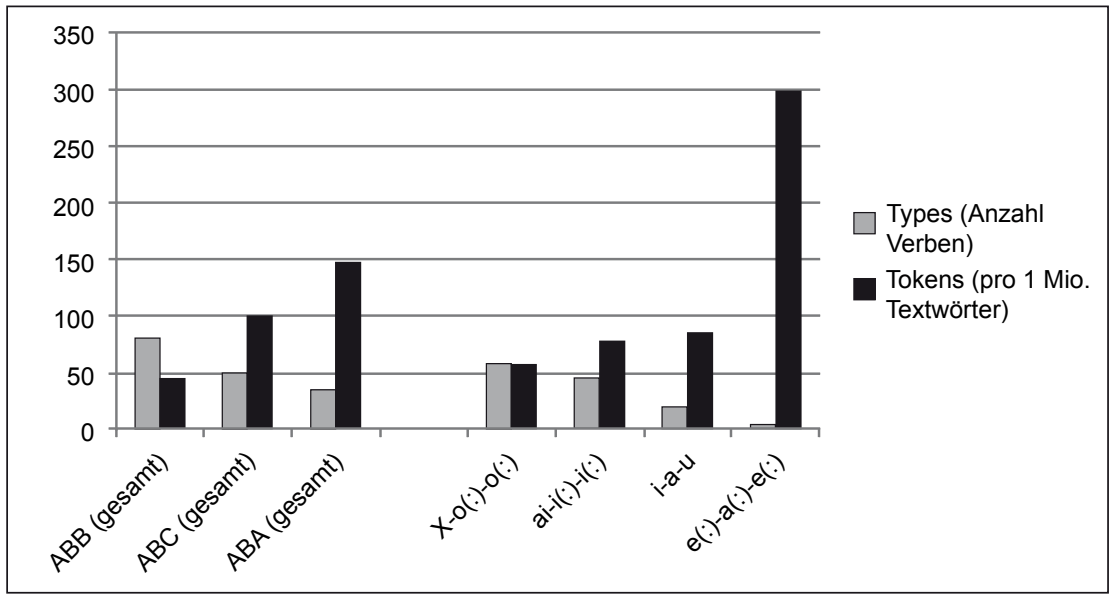

Abb. 4: Type-Token-Verhältnis der verschiedenen Distinktionstypen (links) und ausgewählter Ablautmuster (rechts) $)^{7}$

Unter den Alternanzmustern des Typs ABB ist X-o-o mit seinem ausgewogenen Type-Token-Verhältnis besonders interessant. An der diachronen Entwicklung dieses Musters zeigt sich eindrücklich, dass der Ablaut auch im Nhd. kein Fossil ist. Diese vereinfachte Alternanz ist bis heute semiproduktiv, wie die folgenden Beispiele lektorierter Zeitungstexte mit o- statt a-Ablaut zeigen:

Er schwomm über 400 Meter Freistil zu Gold - und war unglücklich. (FAZ, 11.12.2008)

Gisela Kaiser und ihre Freundinnen sponnen Schafswolle zu Garn.

(WAZ, 12.1.2009)

Sie [...] verweisen auf die Genfer Seerechtskonvention, die das Mittlinienprinzip für Grenzzwiste empfohl. (FR 1993)

Erst nach dem Rückstand besonn sich die Heimelf und erzielte durch einen Doppelschlag den Ausgleich. (Nürnberger Zeitung, 10.3.2008, S. 29)

Die Führung übt zu wenig Druck aus“, ronn es ihr dominahaft aus der Feder. (TAZ 1991)

Es gibt allerdings auch Kritik an dem Projekt: Prozessor-Hersteller Intel etwa scholt den 100-Dollar-Laptop als „Gadget“, also ein technisches Spielzeug. (Hannoversche Allgemeine, 27.9.2007, S. 14)

Das Muster ist eine fnhd. Innovation; der Anteil von Verben mit $o$ in Prät. und Part. II steigt in dieser Phase von lautgesetzlich 14\% durch morphologische Generalisierung auf 28\% der starken Verben (Dammers/Hoffmann/ Solms 1988, S. 538). Das Kerngebiet der Innovation ist das (Ost-)Mittel-

Zahlen nach Nowak (i.Vorb.): Types beziehen sich auf die Mitgliederzahl eines Musters, Tokens auf die durchschnittliche Gebrauchsfrequenz (Simplizia) pro 1 Mio. Textwörter, Datenbasis: Leipziger Projekt Wortschatz (Stand 2011, ca. 217 Mio. Wörter). 
deutsche, wo das Muster durch Lautwandel gestützt ist (mitteldt. Senkung $u>$ o). Auf der Basis des Bonner Fnhd.-Korpus wurde das Phänomen von Solms (1984) an Texten untersucht. Nowak (2010 und i.Vorb.) erforscht X-o-o an einem diachronen Korpus von ca. 70 Grammatiken (16.-21. Jahrhundert), wobei sie auch den Vergleich zum Niederländischen und Luxemburgischen einbezieht, die ähnliche Übergeneralisierungen zeigen.

Bei X-o-o handelt es sich um keine gewöhnliche Ablautreihe, da der Infinitivvokal hier unspezifiziert ist (daher $\underline{X}-0-0)$. Wir haben es also nicht mit einer Ableitungsbeziehung, einer so genannten inputorientierten Generalisierung zu tun wie z.B. bei der Gruppe um reiten mit „wenn ei im Inf., dann $i$ in Prät. und Part. II“ der Fall, sondern mit einer Wohlgeformtheitsbedingung für abgeleitete Formen (outputorientierte Generalisierung: Prät., Part. II o). Dennoch verhält sich das Muster wesentlich systematischer als etwa die traditionelle Ablautreihe VII, deren Mitglieder verschiedene Vokale in Präsens und Part. II aufweisen $(a, a u, o, u)$ und als einzige Gemeinsamkeit $i$ : im Prät. teilen. D.h., wenn man für das Nhd. überhaupt noch von Ablautreihen sprechen will, kann man wie Nowak (2010) X-o-o mit vollem Recht als „8. Ablautreihe“ aufnehmen.

Heute gibt es knapp 50 Verben mit o-o in Prät. und Part. II. Davon haben 20 das Muster sekundär durch morphologischen Ausgleich erworben. Im Fnhd. wurde X-o-o über den heutigen Stand hinaus auf weitere 29 Verben generalisiert (Nowak 2010, S. 435), auch die oben an Zeitungsbelegen illustrierten Schwankungen lassen sich bereits im Fnhd. belegen. Die Verben in Tabelle 5 gehören entweder heute noch zu X-o-o (unterstrichen) oder haben vorübergehend zu diesem Muster tendiert.

\begin{tabular}{|c|c|c|}
\hline \multicolumn{2}{|c|}{ hist. Ablautreihe } & Mitglieder der „8. Ablautreihe“ \\
\hline IIIa & $\mathrm{i}-\mathrm{a} / \mathrm{u}-\mathrm{O}^{8}$ & $\begin{array}{l}\text { glimmen, klimmen ; fnhd. Variation: beginnen, gewinnen, bis heute } \\
\text { Variation: rimnen, schwimmen, spinnen, besinnen }\end{array}$ \\
\hline $\mathrm{IIIb}$ & $\mathrm{e}-\mathrm{a} / \mathrm{u}-\mathrm{O}$ & 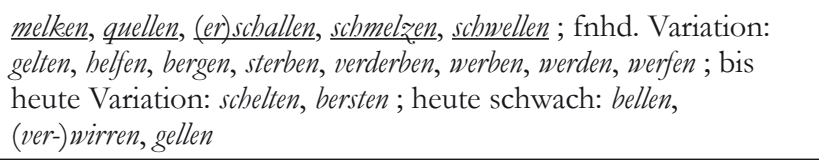 \\
\hline IV & $\mathrm{e}-\mathrm{a} / \hat{\mathrm{a}}-\mathrm{O}$ & $\begin{array}{l}\text { dreschen, fechten, flechten, (er) löschen, scheren; fnhd. Variation: brechen, } \\
\text { gebären, nehmen, schrecken, stechen, stehlen, treffen; heute Variation: } \\
\text { be-/empfeblen; heute schwach: rächen, schwären, verheblen }\end{array}$ \\
\hline $\mathrm{V}$ & $e-a / \hat{a}=e$ & gären, weben, heute schwach: pflegen, bewegen, wägen \\
\hline VI & $\mathrm{V}-\mathrm{u}=\mathrm{V}$ & beben, schwören \\
\hline VII & $\mathrm{V}-\mathrm{ie}-\mathrm{V}$ & Fnhd. Variation: laufen \\
\hline
\end{tabular}

Tab. 5: Gegenwärtige und ehemalige Mitglieder der „8. Ablautreihe“ (nach Nowak 2010, i.Vorb.)

$8 \quad$ Mit Senkung $u>o$ vor Doppelnasal, sonst gilt bei Reihe IIIa $u$ im Part. II. Normalerweise wären die links angegebenen Alternanzmuster (Inf. - Prät.Sg/Pl. - Part. II) zu erwarten; die durchgestrichenen Vokale wurden durch $o$ verdrängt. 
Hier fällt zum einen auf, dass für viele der Verben die Annahme von $o$ eine Zwischenstation zum schwachen Verb war, etwa für bellen und derzeit melken, worauf Grimm im eingangs angeführten Zitat anspielt (Unterstreichung AD): „fühlt man aber nicht, dasz es schöner und deutscher klinge zu sagen buk wob boll (früher noch besser wab ball) als backte webte bellte, und dasz zu jener form die participia gebacken gewoben gebollen stimmen?" Für andere Verben erweist sich X-o-o dagegen als stabile Auffangklasse, z.B. für schmelzen, scheren oder quellen, das Popowitsch $(1754$, S. 348) hier in seinem Schwankungsstadium aufgreift:

Quellen, du quillst, es quillt, es quall (Böd. Steinb. Smotth. [sic]), es quälle [...]; es ist gequollen, quill. So gehet auch verquellen, z.B. die Tbür ist verquollen. Frisch und Hr. Gottsched schreiben quoll, anstatt quall.

Zum anderen fällt auf, dass die meisten Verben nicht zum zentralen Wortschatz gehören, dass es sich um wenig frequente bzw. an Frequenz einbüBende Verben handelt (Zahlen dazu in Nowak 2010 und i.Vorb.). Die Generalisierung über den nhd. Stand hinaus (z.B. auf befeblen, steblen, sinnen, werben) klingt in Texten bereits im 17. Jahrhundert wieder ab, in Grammatiken erreicht sie erst im 18. Jahrhundert ihren Höhepunkt (Nowak i.Vorb.). Hier bestätigt sich erneut, dass Kodifizierer auf Sprachwandel reagieren, ihn aber nur in Ausnahmefällen bedingen.

Wie muss man sich nun die Generalisierung von X-o-o vorstellen? Der Vokal $o$ hatte mit der mitgliederstarken Ablautreihe II bereits eine solide Basis im Ablautsystem. Im Part. II war er zusätzlich in weiteren Reihen (III und IV) vertreten, siehe Tabelle 6:

\begin{tabular}{l|l|l|l|l|l}
\multirow{2}{*}{ AL-Reihe } & Inf., Präs. & Prät.Sg. & Prät.Pl. & Part. II & Distinktionstyp \\
\hline \multirow{2}{*}{ I } & rîten & reit & riten & geritten & \\
\hline \multirow{2}{*}{ II } & bieten & bôt & buten & geboten & ABB \\
\cline { 2 - 5 } & fliegen & flouc & flugen & geflogen & \\
\hline \multirow{2}{*}{ III } & finden & fant & funden & gefunden & \multirow{2}{*}{ ABC } \\
\cline { 2 - 5 } & belfen & balf & bulfen & geholfen & \\
\hline \multirow{2}{*}{ IV } & nemen & nam & nâmen & genomen & \\
\hline V & geben & gap & gâben & gegeben & \\
\hline VI & graben & gruop & gruoben & gegraben & ABA \\
\hline VII & slâfen & slief & sliefen & geslâfen &
\end{tabular}

Tab. 6: Basis von X-o-o im Ablautsystem des Mittelhochdeutschen 
Nun nahm, wie bereits beim präteritalen Numerusausgleich angesprochen, in der fnhd. Phase die Frequenz des Perfekts auf Kosten des Präteritums zu. Man kann davon ausgehen, dass dadurch die Partizipien im mentalen Lexikon der Sprecher/innen besser verankert waren als die Präteritalformen (vgl. Solms 1984, S. 319). Bei Verben, die an Gebrauchsfrequenz einbüßten und die eine lautgesetzliche Basis für $o$ im eigenen Paradigma hatten, konnte sich dieses $o$ vom Part. II auf das Prät. ausbreiten (intraparadigmatische Generalisierung, z.B. bei melkenn). Bei frequenteren Verben wird das Prät. zu gut memoriert (belfen), hier greift nur der präteritale Numerusausgleich:

Ablautreihe IIIb: $\begin{array}{l}\text { belfen } \\ \text { melken }\end{array}$ molf(en $)$ - geholfen
gemolken

Spektakulärer ist die Entwicklung bei Verben, die im eigenen Paradigma überhaupt keine Basis für $o$ haben, z.B. weben, das sich normalerweise wie geben verhalten sollte.

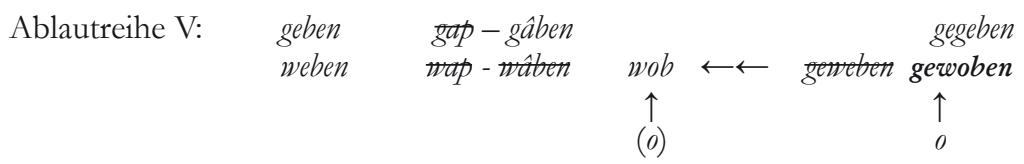

Hier muss der Vokal o über eine interparadigmatische Generalisierung ins Paradigma gelangt sein, d.h. die Sprecher/innen haben das Alternanzen übergreifende Muster X-o-o erkannt und produktiv angewendet. Dabei lässt sich für das Fnhd. eine Staffelung der Annahme von o beobachten. Wenn noch nicht vorhanden, tritt es zuerst im Part. II auf, dann erst im Präteritum, dort zuerst im Plural, dann im Singular (Ebert et al. 1993, S. 273; Nowak 2010, S. 433). Das spricht für ein Ineinandergreifen von zuerst inter- und dann intraparadigmatischer Generalisierung, wie bei weben durch die Pfeile angedeutet.

Im Konjunktiv II (Konj. II) entwickeln sich auf der Basis des neuen Präteritalvokals $o$ Formen mit $\ddot{o}$, und zwar auch bei Verben, bei denen sich später wieder $a$ im Prät. durchgesetzt hat, die also nur vorübergehend zu X-o-o tendierten. Bei diesen Verben erreichen die ö-Konjunktive in Grammatiken ihre Hochphase erst mit dem Abklingen der indikativischen 0 -Formen ab dem 18. Jahrhundert (Nowak i.Vorb.), also dann, als im Indikativ wieder a gilt. Sie zeigen - oft bis heute - Variation im Konj. II, weil sich einerseits der alte Konjunktivvokal $\ddot{o}$ gehalten hat, zusätzlich aber ein neuer Konjunktiv mit $\ddot{a}$ zum neuen Präteritalstamm auf a gebildet wurde:

$\begin{array}{llll} & \text { alt } & & \text { neu } \\ \text { begann }- & \text { begönne } & \text { oder } & \text { begänne? } \\ \text { gewann - } & \text { gewönne } & \text { oder } & \text { gewänne? }\end{array}$


Bei einer Präferenz der $\ddot{o}$-Formen setzt sich eine Strategie morphologischer Kontrasterhöhung durch (vgl. Lotze/Gallmann 2009), da ö-Formen bei Präsensvokal $e$ distinktiver sind, z.B. steble - stähle aber stöble. Sprachkritiker wie Wustmann (1896, S. 59) propagieren den Erhalt der $\ddot{o}$-Formen allerdings weniger mit Systembezug als mit dem bereits angesprochenen Topos konservativ $=$ hochwertig:

Bei einigen Verben, wie bei beginnen, hat der Streit zwischen $\ddot{a}$ und $\ddot{u}$ im Anschluss an das $o$ des Partizips (begonnen) $\ddot{o}$ in Aufnahme gebracht. Auch hier verdienen diese Formen mit ö (beföble, begönne, besönne, empföhle, gewönne, gölte, rönne, schölte, schwömme, spönne), da sie den Formen mit umgewandeltem Pluralvokal entsprechen, den Vorzug vor denen mit $\ddot{a}$.

Nowak (i.Vorb.) zeigt, dass sich Konj. II mit $\ddot{o}$ als Varianten zu $\ddot{a}$ bis in heutige Grammatiken halten, wobei $\ddot{a}$ zunehmend als Leitvariante ausgezeichnet wird. Dies lässt sich als Optimierung der Morphemkonstanz zwischen Prät. Ind. und Konj. II auf Kosten der Modus-Distinktivität interpretieren: steble - stabl - stöble > steble - stạbl - stäble. Dass sich die Variation hier so lange hält, erklärt sich also auch aus dem Konflikt der beiden lokalen Optimierungen Distinktivität vs. Morphemkonstanz (siehe auch Eisenberg 1997).

Warum wurde nun gerade das Muster X-o-o über starke Subklassen hinweg generalisiert? Wir haben gesehen, dass $o$ eine breite Ausgangsbasis im Ablautsystem hatte und dass dort, wo bereits $o$ im Part. II galt, die Generalisierung ins Prät. häufiger eintrat. Für das mitteldt. Sprachgebiet kommt als phonologischer Faktor die Senkung $u>o$ begünstigend hinzu (vor Nasal [dialektal auch Liquid], z.B. mhd. sunne $>$ nhd. Sonne), die bei Verben der Reihe III und IV auch im Prät.Pl. zu o-Formen führen konnte (Ebert et al. 1993, Kap. III.6.7). Begünstigend wirkt auch die Signalstärke des Vokals $o$ im Ablautsystem: $o$ kommt kaum als Präsens- und Infinitivvokal vor und ist damit ein besonders eindeutiger Marker für Prät. und Part. II (Nowak i.Vorb.; zur nicht zufälligen Verteilung von Vokalqualitäten im nhd. Ablautsystem vgl. auch Wiese 2008). Als wichtiger gebrauchsbedingter Faktor, aus dem sich auf den Grad an Verankerung im mentalen Lexikon schließen lässt, hat sich eine niedrige bzw. abnehmende Frequenz der betroffenen Verben erwiesen (Hempen 1988, S. 317). Deutlich wird das daran, dass sich Verben unterschiedlicher Frequenzbereiche bei gleicher phonologischer Struktur divergent verhalten: Während minderfrequentes glimmen schon seit dem Fnhd. o-Formen aufweist, schwanken wir heute noch bei spinnen (spann/sponn) und würden bei höherfrequenten Verben wie beginnen und gewinnen nicht auf die Idee kommen, * sie gewonn oder *begonn zu bilden. Hier sind die Präteritalformen zu gut durch häufigen Gebrauch verankert. Das zeigt eindrücklich, dass es sich um morphologischen Ausgleich handelt, nicht um phonologischen Wandel (siehe Nowak 2010, S. 436; i.Vorb. für diachrone Frequenzangaben). 
X-o-o ist also eine teilregularisierte Auffangklasse für „schwächelnde“ starke Verben, die eine Zwischenstufe im Übergang zur schwachen Flexion bilden kann (z.B. bellen, melken), seit der fnhd. Phase aber auch 20 Verben dauerhaft in der starken Flexion stabilisiert. Zweifelsfälle wie spinnen und schwimmen zeigen, dass die „8. Ablautreihe“ noch heute produktiv ist. Auch sprachspielerisch, etwa in der Lyrikabteilung der „Gesellschaft zur Stärkung der Verben“, wird besonders das X-o-o-Muster übergeneralisiert:

Ein Schwachverbenstärker aus Labenz/ stork Verben von morgens bis abends.

Auch Nomen er stork, wobei er bemork/ die Einspar so manchen Buchstabens. (http://verben.texttheater.net/Drei_Limericks; 2.7.2013)

X-o-o ist ein Pfad der Regularisierung konkreter Vokalalternanzen, den man in den abstrakten Regularisierungspfad aus Abbildung 3 einbetten kann. Wichtigste Bedingung ist geringe/fallende Tokenfrequenz, die zu schlechter Verankerung der Prät.-Formen und deren Neubildung auf Basis des eigenen Part. II oder anderer Verben mit X-o-o führt.

\section{Fazit}

Insgesamt zeigen die hier dargestellten Entwicklungen, dass der Umbau von Ablautmustern prinzipiengesteuert ist: Starke Verben sind keine morphologischen Fossilien, sondern werden bis heute reorganisiert und unterliegen Subregularitäten wie X-o-o. Aus der Langzeitperspektive des Systems stirbt Ablaut nicht (nur) aus, sondern er kommt im System an: Er beginnt als Stammalternation in der Phonetik (Voride.), wird dann phonologisiert (Ide.) und übernimmt allmählich grammatische Aufgaben (Germ.), ähnlich wie später auch der Umlaut. Das Deutsche erhält im Vergleich germanischer Sprachen eine hohe Anzahl starker Verben - trotz ihrer komplexen Bildungsweise. Umschichtungen innerhalb des Ablautsystems führen zu einer frequenzbasierten Verteilung: Häufiger Gebrauch begünstigt Erhalt, Irregularisierung und Vereinzelung, verminderter Gebrauch den Anschluss an vereinfachte Ablautmuster in Großgruppen (X-o-o) oder den vollen Übergang in die schwache Flexion. Tokenfrequenz ist aber nicht alles: Die Zugehörigkeit zu mitgliederstarken Gruppen mit charakteristischen phonologischen Strukturen wie $i+$ Nasal + Konsonsonant (singen), besonders wenn diese bei schwachen Verben selten sind („Inseln der Verlässlichkeit“, vgl. Mailhammer 2007b), kann auch minderfrequente Mitglieder stark halten. J. Grimm hat in seinem eingangs zitierten Plädoyer also - auch - recht: Ablaut ist im Deutschen heute noch aktiver als man auf den ersten Blick vermutet.

Mit diachroner Tiefe zeigt sich: Phänomene, die als rezente Anzeichen von Sprachverfall gehandelt werden, sind Teil sehr systematischer und sich über einen langen Zeitraum erstreckender Umbauprozesse. Ihnen liegen 
systemlinguistische, kognitive und frequenzielle Bedürfnisse der Sprecher/ innen zugrunde, auf die Kodifizierer und Sprachkritiker nur geringen Einfluss haben. Diese Perspektive auch für Laien zu öffnen, wäre eine lohnende Aufgabe für die historische Linguistik.

\section{Literatur}

Adelung, Johann Chr. (1782): Umständliches Lehrgebäude der deutschen Sprache. Bd. 1. Leipzig.

Augst, Gerhard (1975): Wie stark sind die starken Verben? Überlegungen zur Subklassifikation der nhd. Verben. In: Augst, Gerhard: Untersuchungen zum Morpheminventar der deutschen Gegenwartssprache. (= Forschungsberichte des Instituts für deutsche Sprache 25). Tübingen, S. 231-281.

Best, Karl-Heinz (2003): Spracherwerb, Sprachwandel und Wortschatzwachstum in Texten. Zur Reichweite des Piotrowski-Gesetzes. In: Glottometrics 6, S. 9-34.

Bittner, Andreas (1996): Starke, schwache' Verben und schwache ,starke ${ }^{6}$ Verben. Deutsche Verbflexion und Natürlichkeit. (= Studien zur deutschen Grammatik 51). Tübingen.

Bybee, Joan L. (1985): Morphology. A study on the relation between meaning and form. (= Typological Studies in Language 9). Amsterdam/Philadelphia.

Clahsen, Harald et al. (2002): Strong stems in the German mental lexicon: Evidence from child language acquisition and adult processing. In: Kaufmann, Ingrid/Stiebels, Barbara (Hg.): More than words: A festschrift for Dieter Wunderlich. (= Studia grammatica 53). Berlin, S. 91-112.

Corr, Andreas (2013): Über die konservative Traditionslinie populärer Sprach- und Stilratgeber. In: Sprachreport 1-2, S. 13-18.

Dammel, Antje (2010): Konjugationsklassenwandel. Prinzipien des Ab-, Um- und Ausbaus verbalflexivischer Allomorphie in germanischen Sprachen. (= Studia Linguistica Germanica 103). Berlin/New York.

Dammers, Ulf/Hoffmann, Walter/Solms, Hans-Joachim (1988): Grammatik des Frühneuhochdeutschen. Bd. 4: Flexion der starken und schwachen Verben. Heidelberg.

Dentler, Sigrid (1998): Gab es den Präteritumschwund? In: Askedal, John Ole (Hg.): Historische germanische und deutsche Syntax. (= Osloer Beiträge zur Germanistik 21). Frankfurt a.M., S. 133-147.

Duden (1998): Der Duden. Bd. 4: Grammatik der deutschen Gegenwartssprache. 6., neu bearb. Aufl. Hrsg. v. d. Dudenredaktion. Mannheim u.a.

Durrell, Martin (2001): Strong verb Ablaut in the West Germanic languages. In: Watts, Sheila/West, Jonathan/Solms, Hans-Joachim (Hg.): Zur Verbmorphologie germanischer Sprachen. (= Linguistische Arbeiten 446). Tübingen, S. 5-18.

Ebert, Robert Peter et al. (1993): Frühneuhochdeutsche Grammatik. (= Sammlung kurzer Grammatiken germanischer Dialekte: A, Hauptreihe 12). Tübingen. 
Eisenberg, Peter (1997): Konjunktiv als Flexionskategorie im heutigen Deutsch. In: Debus, Friedhelm/Leirbukt, Oddleif (Hg.): Aspekte der Modalität im Deutschen. (= Germanistische Linguistik 136). Hildesheim u.a., S. 37-56.

Enger, Hans-Olav (1994): A possible constraint on non-affixal inflection. In: Lingua 114, S. $59-75$.

Faust, Manfred (1980): Morphologische Regularisierung in Sprachwandel und Spracherwerb. In: Folia Linguistica XIV, 3-4, S. 387-411.

Grimm, Jacob ([1847] 1864): Über das Pedantische in der deutschen Sprache. In: Grimm, Jacob: Kleinere Schriften. 1. Bd. Berlin, S. 327-373.

Gueintz, Christian (1978 [1641]): Deutscher Sprachlehre Entwurf. Hildesheim. [Nachdruck d. Ausg. Köthen 1641.]

Hempen, Ute (1988): Die starken Verben im Deutschen und Niederländischen. Diachrone Morphologie. (= Linguistische Arbeiten 214). Tübingen.

Hundt, Markus (2009): Normverletzungen und neue Normen. In: Konopka/Strecker (Hg.), S. 117-140.

Klein, Wolf-Peter (2011): Sprachliche Zweifelsfälle im Frühneuhochdeutschen. Alte und neue Perspektiven ihrer Erforschung. In: Lobenstein-Reichmann, Anja/Reichmann, Oskar (Hg.): Frühneuhochdeutsch. Aufgaben und Probleme seiner linguistischen Beschreibung. (= Germanistische Linguistik 213/215). Hildesheim u.a., S. 275316.

Konopka, Manfred/Strecker, Bruno (Hg.) (2009): Deutsche Grammatik - Regeln, Normen, Sprachgebrauch. (= Jahrbuch des Instituts für Deutsche Sprache 2008). Berlin/New York.

Lotze, Stefan/Gallmann, Peter (2009): Norm und Variation beim Konjunktiv II. In: Konopka/Strecker (Hg.), S. 222-239.

Mailhammer, Robert (2007a): The Germanic strong verbs. Foundations and development of a new system. (= Trends in Linguistics. Studies and Monographs 183). Berlin/New York.

Mailhammer, Robert (2007b): Islands of resilience: The history of the German strong verbs from a systemic point of view. In: Morphology 17, S. 77-108.

Nowak, Jessica (2010): Im Spannungsfeld zwischen starken und schwachen Verben. Die Entstehung der „8. Ablautreihe“ im Deutschen, Niederländischen und Luxemburgischen. In: Dammel, Antje/Kürschner, Sebastian/Nübling, Damaris (Hg.): Kontrastive germanistische Linguistik. Bd. 2. (= Germanistische Linguistik 206-209). Hildesheim u.a., S. 429-472.

Nowak, Jessica (i.Vorb.): Zur Legitimation einer 8. Ablautreihe - Eine kontrastive Analyse zu ihrer Entstehung im Deutschen, Niederländischen und Luxemburgischen. Diss. Univ. Mainz.

Nübling, Damaris (1998): Wie die Alten sungen ... Zur Rolle von Frequenz und Allomorphie beim präteritalen Numerusausgleich im Frühneuhochdeutschen. In: Zeitschrift für Sprachwissenschaft 17 (1998), S. 185-203. 
Nübling, Damaris/Dammel, Antje (2004): Relevanzgesteuerter morphologischer Umbau im Frühneuhochdeutschen. In: Beiträge zur Geschichte der deutschen Sprache und Literatur 126, S. 177-207.

Popowitsch, Johann Siegmund Valentin (1754): Die nothwendigsten Anfangsgruende der teutschen Sprachkunst zum Gebrauche der oesterreichischen Schulen. Wienn.

Ringe, Donald (2006): A linguistic history of English. Bd. 1: From Proto-Indo-European to Proto-Germanic. Oxford.

Schneider, Wolf (2009): Gewönne doch der Konjunktiv! Sprachwitz in 66 Lektionen. Reinbek b. Hamburg.

Solms, Hans-Joachim (1984): Die morphologischen Veränderungen der Stammvokale der starken Verben im Frühneuhochdeutschen. Untersucht an Texten des 14.-18. Jahrhunderts. Bonn.

Stedje, Astrid (1987): Warum nur im Germanischen? Altes und Neues zum Ablaut der starken Verben. In: Studier i modern språkvetenskap, Ny serie 7, S. 96-113.

Wegera, Klaus-Peter/Waldenberger, Sandra (2012): Deutsch diachron. Eine Einführung in den Sprachwandel des Deutschen. (= Grundlagen der Germanistik 52). Berlin.

Werner, Otmar (1987): The aim of morphological change is a good mixture - not a uniform language type. In: Giacalone Ramat, Anna/Carruba, Onofrio/Bernini, Giuliano (Hg.): Papers from the 7th international Conference of Historical Linguistics. (= Amsterdam Studies in the Theory and History of Linguistic Science: Series IV. Current Issues in Linguistic Theory 48). Amsterdam/Philadelphia, S. 591616.

Wiese, Bernd (2008): Form and function of Verbal Ablaut in Contemporary Standard German. In: Sackmann, Robin (Hg.): Explorations in Integrational Linguistics: Four essays on German, French, and Guarani. Amsterdam, S. 97-152.

Wurzel, Wolfgang Ullrich (1996): Morphologischer Strukturwandel. Typologische Entwicklungen im Deutschen. In: Lang, Ewald/Zifonun, Gisela (Hg.): Deutsch typologisch. (= Jahrbuch des Instituts für deutsche Sprache 1995). Berlin/New York, S. 492-525.

Wustmann, Gustav (1896): Allerhand Sprachdummheiten. Kleine deutsche Grammatik des Zweifelhaften, des Falschen und des Häßlichen. Ein Hilfsbuch für alle, die sich öffentlich der deutschen Sprache bedienen. 2., verb. u. verm. Ausg. Leipzig.

Zuraw, Kie (2003): Probability in language change. In: Bod, Rens/Hay, Jennifer/Jannedy, Stefanie (Hg.): Probabilistic Linguistics. Cambridge, MA u.a., S. 139-176. 